Article

\title{
Nonlocal Torsional Vibration of Elliptical Nanorods with Different Boundary Conditions
}

\author{
Farshad Khosravi ${ }^{1}$, Seyyed Amirhosein Hosseini ${ }^{2}$, Babak Alizadeh Hamidi ${ }^{3}$, \\ Rossana Dimitri ${ }^{4}$ (D) and Francesco Tornabene ${ }^{4, *} \mathbb{D}$ \\ 1 Department of Aerospace Engineering, K.N. Toosi University of Technology, 1996715433 Tehran, Iran; \\ fkhosravi@mail.kntu.ac.ir \\ 2 Department of Industrial, Mechanical and Aerospace Engineering, Buein Zahra Technical University, \\ Buein Zahra, 3451745346 Qazvin, Iran; hosseini@znu.ac.ir \\ 3 Department of Mechanical Engineering, University of Tabriz, 5166614766 Tabriz, Iran; \\ babak.alizade@rocketmail.com \\ 4 Department of Innovation Engineering, University of Salento, 73100 Lecce, Italy; \\ rossana.dimitri@unisalento.it \\ * Correspondence: francesco.tornabene@unisalento.it
}

Received: 17 July 2020; Accepted: 5 August 2020; Published: 7 August 2020

check for updates

\begin{abstract}
This work aims at investigating the free torsional vibration of one-directional nanostructures with an elliptical shape, under different boundary conditions. The equation of motion is derived from Hamilton's principle, where Eringen's nonlocal theory is applied to analyze the small-scale effects. The analytical Galerkin method is employed to rewrite the equation of motion as an ordinary differential equation (ODE). After a preliminary validation check of the proposed formulation, a systematic study investigates the influence of the nonlocal parameters, boundary conditions, geometrical and mechanical parameters on the natural frequency of nanorods; the objective is to provide useful findings for design and optimization purposes of many nanotechnology applications, such as, nanodevices, actuators, sensors, rods, nanocables, and nanostructured aerospace systems.
\end{abstract}

Keywords: carbon nanotubes; elliptical nanorod; free vibration; Hamilton's principle; nonlocal elasticity; torsional vibration

\section{Introduction}

Small-sized structures such as nanorods, nanotubes, nanowires, nanobeams, and nanoshells are increasingly becoming key structural elements in nano- and micro-electromechanical systems (NEMS/MEMS), as well as in nanostructured coatings and materials for aerospace applications. In this context, several theories and experiments have been developed in the literature to study the mechanical, physical, electronic and thermal properties of atomic scaled structures, namely, boron nitride nanotubes [1,2], silica carbide nanotube/wires [3,4], graphene [5,6], and carbon nanotubes (CNTs) [7-10]. Among them, CNTs have been introduced in the late 1990s by lijima [11,12], and have received an increased interest among the scientific community [13-24] due to their outstanding mechanical, thermal, and electrical properties [25-28]. CNTs can be partitioned in two parts, namely, the single-walled carbon nanotube (e.g., SWCNT) and the multi-walled carbon nanotube (e.g., MWCNT) [29]. It is well known that CNTs are hollow tubes rolled up by graphene sheets [11,12], which can be described as one-dimensional structures with lengths much higher than sectional sizes. At the same time, cross-sections are usually modeled with a circular shape, but the possible influence of noncircular shapes on the structural response would be of paramount importance in the design and analysis of many aircraft components and parts [30,31]. In this framework, the classical continuum 
mechanics has largely failed to verify findings at the nanoscale. This has increased the necessity of applying novel continuum mechanics theories and atomic/molecular dynamic simulations, along with the application of many experimental investigations and measurements in line with the high precision of minute sizes, see references [32-37], together with the review papers [38,39].

Starting with the nonlocal elasticity theory proposed for the first time by Eringen [40-44] to handle small-scale effects, many further works have merged nonlocal and gradient continuum formulations to study the size-dependent response of nanorods [45-54], nanobeams [55-63], and nanoplates/nanoshells [64-71]. More specifically, as far as the torsional vibration problem is concerned, different works in the literature have studied the size-dependent torsional behavior of nanorods and CNTs via theoretical and/or numerical nonlocal elasticity formulations. Ansari et al. [72] applied two approaches, namely the strain gradient theory (SGT), and molecular dynamics (MD), to determine the torsional vibration of CNTs, and its sensitivity to the nonlocal parameter. Fatahi-Vajari and Imam [73] investigated the torsional vibration of SWCNTs with zigzag and armchair structures by means of the MD, and surveyed the influence of the small-scale parameter, as well as the chiral effects on the structural response. In addition, a double CNT system connected by a torsional elastic medium was modeled by Arda and Aydogdu [74], for two various boundary conditions (BCs), to illustrate the effect of the elastic medium stiffness and the small-scale parameter on the torsional vibration of the nanostructure. Li [75] applied two reverse nonlocal models, including weakened and enhanced models to justify the nonlocal torsional natural frequencies of CNTs, and proposed a semi-continuum model with discrete atomic layers in the CNT cross section. In a more recent work, Aydogdu and Arda [76] analyzed the torsional frequencies of double carbon nanotubes, based on a nonlocal elasticity theory, while discussing the sensitivity of the response to the nonlocal parameter, the Van der Waals force interaction, and the nanotube length. Demir and Civalek [77] investigated both the torsional and axial vibration of microtubules based on a nonlocal continuous and discrete size-dependent rod model. In line with the previous work, Murmu et al. [78] performed a size-dependent parametric analysis of the torsional vibration of a single-walled CNT-buckyball system for nanoresonators, while tuning the mass inertia for design purposes. Suzuki et al. [79] proposed an ultra-precision sculpturing method in micro- and nano-structures for difficult-to-cut materials. More specifically, elliptical vibration cutting (EVC) was applied with a single crystal diamond as controlled cutting and machining tool for steel materials, while monitoring the structural vibration amplitudes. Similarly, Zhang et al. [80] applied the EVC technology to simplify the machining process of textured surfaces. Different EVC devices were employed, compared and verified in terms of their accuracy; the role of the elliptical vibrator was explained in detail for the manufacturing process of micro- and nano-structures. An elliptical vibration texturing was also adopted by Yang et al. [81] to colorize metallic surfaces with periodic micro- and nano-scale materials, and to optimize the metal surfaces properties.

Based on the above-mentioned practical problems, even more accurate methods have been adopted very recently to treat torsional vibrations, both analytically and/or numerically. In this framework, Mikeš and Jirásek [82] established a numerical method for free warping structures with an elliptical cross section, while adopting the finite element approach to handle more complicated cross sections. Barr [83] studied the torsional vibration of a uniform rod with a rectangular cross section to investigate the dispersion of torsional waves within rods, and its sensitivity to longitudinal stresses and inertia effects. In addition, Stephen [84] modeled beams with an elliptical cross section, and compared the dynamic torsional predictions by different theories. Francu et al. [85] employed the Airy functions to analyze the stress-strain analytical expressions of warping beams with different noncircular cross sections. Christides and Barr [86] modeled one or more pairs of symmetric cracks in uniform beams with a noncircular cross section, for the study of their torsional vibration, and the computation of their fundamental natural frequencies for different crack depths. In some further works by Loya et al. [87-89], different nonlocal cracked models were proposed to treat the torsional and bending vibration of CNTs, and their sensitivity to the nonlocal small-scale parameter, crack severity, and cracked section position, together with different boundary conditions. 
According to the above literature review, however, there is a general lack of works focusing on the nonlocal dynamic torsion of nanostructures with noncircular cross sections. This topic is tackled here for elliptical nanorods in a nonlocal context, as a pioneering study of additional and more complicated shapes of practical interest. The nonlocal governing equation of the problem is obtained by means of Hamilton's principle, and discretized via the Galerkin method. A systematic investigation aims at checking the sensitivity of the nonlocal dynamic twisting response to the nonlocal parameter, geometry parameters, and stiffness of the torsional spring, which could be of great interest for nanostructural design.

\section{Nonlocal Elasticity Theory}

According to the well-known MEMS/NEMS applications of micro/nano-roads under a torsional load (e.g., NEMS oscillators, torsional micromirrors, torsional miroscanners, etc.), nanostructures usually feature noncircular cross sections, primarily of elliptical shape, as visible in the TEM image of Figure 1, for a platinum nanowire on a $\mathrm{MgO}(110)$ substrate [90].

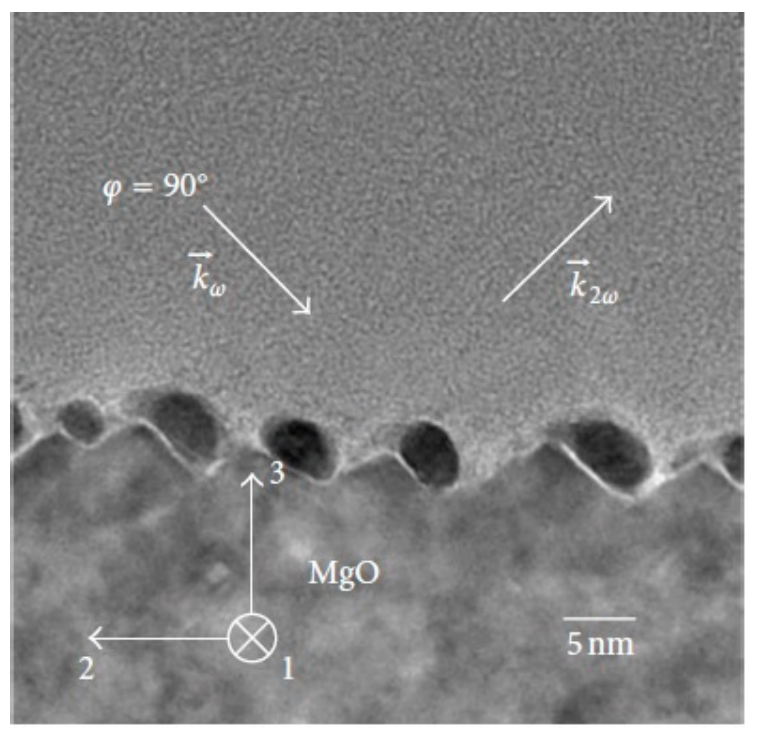

Figure 1. TEM image of a platinum nanowire with an elliptical cross section on the MgO (110) substrates [90].

Let us consider a clamped-clamped (C-C), clamped-free (C-F), and clamped-torsional spring (C-T) nanorod, of length $l$, and radii $a$ and $b$ in the $y$-axis and $z$-axis, respectively, as depicted in Figures 2-4. The torsional spring in Figure 4 , is also denoted as $k_{b}$.

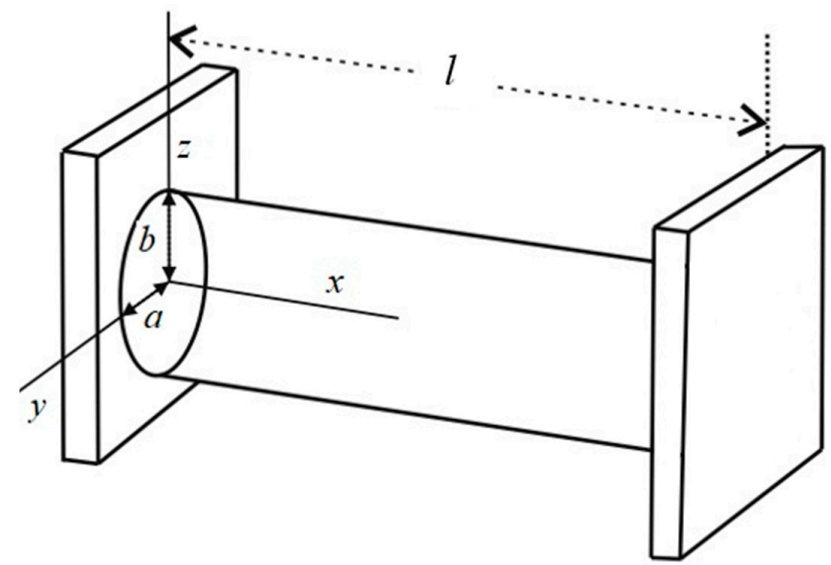

Figure 2. Clamped-clamped (C-C) nanorod with an elliptical cross section. 


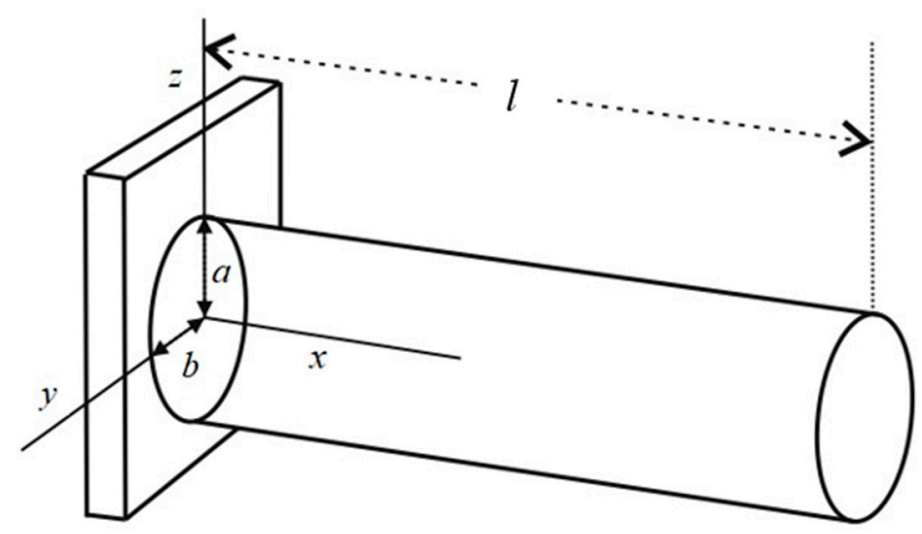

Figure 3. Clamped-free (C-F) nanorod with an elliptical cross section.

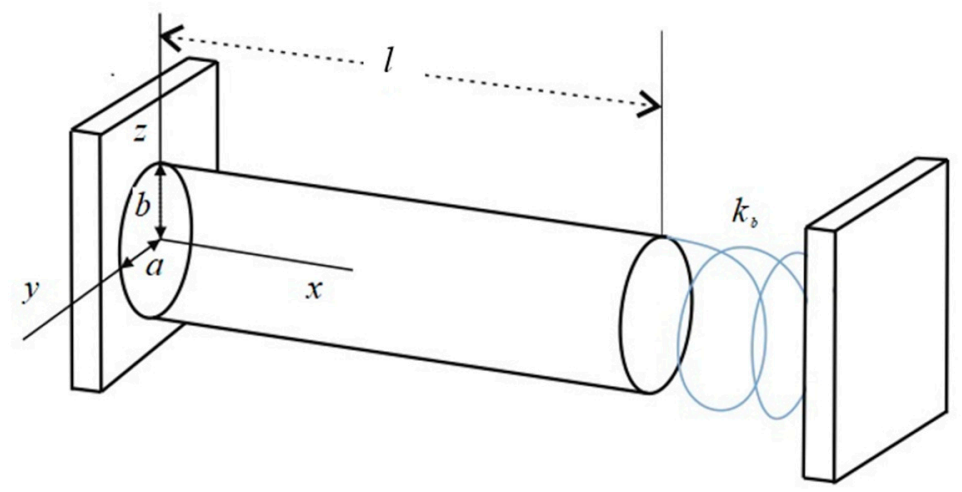

Figure 4. Clamped-torsional (C-T) spring nanorod with an elliptical cross section.

The displacement field for any arbitrary point under a torsional vibration, is defined by the following components

$$
\begin{aligned}
& u(x, t)=\psi(y, z) \frac{\partial \theta}{\partial x} \\
& v(x, t)=-z \theta(x, t) \\
& w(x, t)=y \theta(x, t)
\end{aligned}
$$

where the $x$-axis corresponds to the centerline of the CNT, $u, v$, and $w$ denote the displacement components in the $x, y$ and $z$ direction, respectively. Besides, $\theta$ is the angular twist, and $\psi(y, z)$ represents the warping function, defined as $\psi(y, z)=y \times z\left(\frac{b^{2}-a^{2}}{b^{2}+a^{2}}\right)$. The strain field is, thus, governed by the following kinematic relations

$$
\begin{aligned}
& \varepsilon_{x y}=\frac{\partial u}{\partial y}+\frac{\partial v}{\partial x}=\left(\frac{\partial \psi}{\partial y}-z\right) \frac{\partial \theta}{\partial x} \\
& \varepsilon_{x z}=\frac{\partial u}{\partial z}+\frac{\partial w}{\partial x}=\left(\frac{\partial \psi}{\partial z}+y\right) \frac{\partial \theta}{\partial x} \\
& \varepsilon_{y z}=\frac{\partial v}{\partial z}+\frac{\partial w}{\partial y}=0 \\
& \varepsilon_{x x}=\varepsilon_{y y}=\varepsilon_{z z}=0
\end{aligned}
$$

which are, in turn, related to the stress components $\sigma_{x y}, \sigma_{x z}$ by means of the constitutive relations. The torsional couple acting on the twisted noncircular body is, thus, expressed as [91]

$$
M=\int_{A}\left(\sigma_{x y}\left(\frac{\partial \psi}{\partial y}-z\right)+\sigma_{x z}\left(\frac{\partial \psi}{\partial z}+y\right)\right) d A
$$


Hamilton's Principle

Hamilton's principle is then employed to derive the equation of motion in the following form

$$
\int_{0}^{t} \delta\left(U-T+W_{e x t}\right) d t=0
$$

where $U, T$ and $W_{\text {ext }}$ refers to the strain energy, the kinetic energy, and the external works, respectively. For noncircular nanorods, the strain energy is defined in a variational form as follows

$$
\delta U=\int_{V} \sigma_{i j} \delta \varepsilon_{i j} d V=\int_{V}\left(\sigma_{x y} \delta \varepsilon_{x y}+\sigma_{x z} \delta \varepsilon_{x z}\right) d V
$$

which is combined to Equation (2) to yield

$$
\begin{gathered}
\delta U=\delta \int_{V}\left(\sigma_{x y}\left(\frac{\partial \psi}{\partial y}-z\right) \frac{\partial \theta}{\partial x}+\sigma_{x z}\left(\frac{\partial \psi}{\partial z}+y\right) \frac{\partial \theta}{\partial x}\right) d V \\
=\delta \int_{V}\left(\left(y \sigma_{x z}-z \sigma_{x y}\right)+\left(\sigma_{x y} \frac{\partial \psi}{\partial y}+\sigma_{x z} \frac{\partial \psi}{\partial z}\right)\right) \frac{\partial \theta}{\partial x} d A d x
\end{gathered}
$$

By a further combination of Equation (6) with Equation (3) we obtain the following variational expression for the strain energy

$$
\begin{aligned}
& \delta U=\int_{0}^{l} \int_{A}\left(\left(y \sigma_{x z}-z \sigma_{x y}+\sigma_{x y} \frac{\partial \psi}{\partial y}+\sigma_{x z} \frac{\partial \psi}{\partial z}\right) \frac{\partial \delta \theta}{\partial x}\right) d x d A+ \\
& \left.+\int_{0}^{l} \int_{A}\left(\sigma_{x y} \frac{\partial \delta \psi}{\partial y}+\sigma_{x z} \frac{\partial \delta \psi}{\partial z}\right) \frac{\partial \theta}{\partial x}\right) d x d A
\end{aligned}
$$

By integration of $\delta U$ within a certain lapse of time $[0, t]$ we obtain

$$
\int_{0}^{t} \delta U=-\int_{0}^{t}\left(\int_{0}^{l} \frac{\partial M}{\partial x} \delta \theta d x\right) d t
$$

The kinetic energy is defined as

$$
T=\frac{1}{2} \int_{V} \rho\left[\left(\frac{\partial u}{\partial t}\right)^{2}+\left(\frac{\partial v}{\partial t}\right)^{2}+\left(\frac{\partial w}{\partial t}\right)^{2}\right] d V
$$

By substituting the first derivative of Equation (1) with respect to the time into Equation (9), the kinetic energy can be rewritten as

$$
T=\frac{1}{2} \int_{V} \rho\left[\psi^{2}(y, z)\left(\frac{\partial^{2} \theta}{\partial x \partial t}\right)^{2}+\left(-z \frac{\partial \theta}{\partial t}\right)^{2}+\left(y \frac{\partial \theta}{\partial t}\right)^{2}\right] d V=I_{1}+I_{2}
$$

where $I_{1}$ and $I_{2}$ include the axial and polar inertia terms, defined as

$$
\begin{gathered}
I_{1}=\frac{1}{2} \rho \int_{0}^{l} \int_{A} \psi^{2}(y, z)\left(\frac{\partial^{2} \theta}{\partial x \partial t}\right)^{2} d A d x \\
I_{2}=\frac{1}{2} \rho I_{p} \int_{0}^{l}\left(\frac{\partial \theta}{\partial t}\right)^{2} d x=\frac{1}{2} I_{0} \int_{0}^{l}\left(\frac{\partial \theta}{\partial t}\right)^{2} d x
\end{gathered}
$$


$I_{p}$ and $I_{0}$ denote the polar moment of inertia, and mass inertia, respectively, expressed as

$$
\begin{gathered}
I_{p}=\int_{A}\left(y^{2}+z^{2}\right) d A \\
I_{0}=\rho I_{p}
\end{gathered}
$$

The first variation of $I_{1}$ can be determined as follows

$$
\delta I_{1}=\int_{0}^{l} \rho I_{\psi} \frac{\partial^{2} \theta}{\partial t \partial x} \frac{\partial^{2}(\delta \theta)}{\partial t \partial x} d x+\int_{0}^{l} \rho I_{\theta}^{2} \int_{A} \psi \delta \psi d A
$$

where

$$
\begin{gathered}
I_{\psi}=\int_{A} \psi^{2} d A \\
I_{\theta}=\int_{0}^{l} \rho\left(\frac{\partial^{2} \theta}{\partial t \partial x}\right) d x
\end{gathered}
$$

The first variation of $I_{1}$ in the Hamilton's principle can be expressed as

$$
\int_{0}^{t} \delta I_{1}=\rho I_{\psi} \int_{0}^{t} \int_{0}^{l} \frac{\partial^{4} \theta}{\partial x^{2} \partial t^{2}} \delta \theta d x d t-\left.\rho I_{\psi} \int_{0}^{t} \frac{\partial^{3} \theta}{\partial x \partial t^{2}} \delta \theta\right|_{0} ^{l} d t
$$

whereas, the first variation of $I_{2}$ is defined as

$$
\delta I_{2}=\int_{0}^{l} \rho I_{p} \frac{\partial \theta}{\partial t} \frac{\partial \delta \theta}{\partial t} d x=I_{0} \int_{0}^{l} \frac{\partial \theta}{\partial t} \frac{\partial \delta \theta}{\partial t} d x
$$

By substitution of $I_{2}$ in Hamilton's principle, we calculate

$$
\int_{0}^{t} \delta I_{2}=-I_{0} \int_{0}^{t} \int_{0}^{l} \frac{\partial^{2} \theta}{\partial t^{2}} d x
$$

Finally, the variation of kinetic energy can be stated as

$$
\begin{aligned}
& \delta \int_{0}^{t} T=\rho I_{\psi} \int_{0}^{t} \int_{0}^{l} \frac{\partial^{4} \theta}{\partial x^{2} \partial t^{2}} \delta \theta d x d t-\left.\rho I_{\psi} \int_{0}^{t} \frac{\partial^{3} \theta}{\partial x \partial t^{2}} \delta \theta\right|_{0} ^{l} d t \\
& +\int_{0}^{t} I_{\theta} \int_{A} \psi \delta \psi d A d t-I_{0} \int_{0}^{t} \int_{0}^{l} \frac{\partial^{2} \theta}{\partial t^{2}} d x
\end{aligned}
$$

In total absence of the external work, the combination of Equations (4), (8), and (21) yields the following equation of motion

$$
\frac{\partial M}{\partial x}=I_{0} \frac{\partial^{2} \theta}{\partial t^{2}}-\rho I_{\psi} \frac{\partial^{4} \theta}{\partial x^{2} \partial t^{2}}
$$

According to Eringen [41], the nonlocal constitutive relations of the nanostructure take the following form

$$
\begin{aligned}
& \sigma_{x y}-\mu^{2} \frac{\partial^{2} \sigma_{x y}}{\partial x^{2}}=G\left(\frac{\partial \psi}{\partial y}-z\right) \frac{\partial \theta}{\partial x} \\
& \sigma_{x z}-\mu^{2} \frac{\partial^{2} \sigma_{x z}}{\partial x^{2}}=G\left(\frac{\partial \psi}{\partial z}+y\right) \frac{\partial \theta}{\partial x}
\end{aligned}
$$


$\mu$ being the nonlocal parameter, which accounts for the characteristic internal length of the covalent bonds of carbon. Thus, the nonlocal twisting moment is governed by the following relation

$$
M-\mu^{2} \frac{\partial^{2} M}{\partial x^{2}}=G \int_{A}\left(\left(\frac{\partial \psi}{\partial y}-z\right)^{2}+\left(\frac{\partial \psi}{\partial z}+y\right)^{2}\right) \frac{\partial \theta}{\partial x} d A=G I_{\Phi} \frac{\partial \theta}{\partial x}
$$

with

$$
I_{\Phi}=\int_{A}\left(\left(\frac{\partial \psi}{\partial y}-z\right)^{2}+\left(\frac{\partial \psi}{\partial z}+y\right)^{2}\right) d A
$$

Substituting the first derivative of Equation (22) into Equation (25) yields

$$
M-\mu^{2}\left(I_{0} \frac{\partial^{3} \theta}{\partial t^{2} \partial x}-\rho I_{\psi} \frac{\partial^{5} \theta}{\partial x^{3} \partial t^{2}}\right)=G I_{\Phi} \frac{\partial \theta}{\partial x}
$$

Thus, the equation of motion for noncircular nanorods, takes the following final form

$$
\rho I_{\psi} \frac{\partial^{4} \theta}{\partial x^{2} \partial t^{2}}-I_{0} \frac{\partial^{2} \theta}{\partial t^{2}}+\mu^{2}\left(I_{0} \frac{\partial^{4} \theta}{\partial t^{2} \partial x^{2}}-\rho I_{\psi} \frac{\partial^{6} \theta}{\partial x^{4} \partial t^{2}}\right)+G I_{\Phi} \frac{\partial^{2} \theta}{\partial x^{2}}=0
$$

\section{Analytical Solution}

Based on the Galerkin method, we compute the theoretical solution of Equation (28), for nanorods with an elliptical cross-section, namely

$$
\theta(x, t)=\sum_{n=1}^{\infty} \Theta_{n}(x) e^{i \omega t}
$$

$\Theta_{n}$ being the $n$th mode shape, for a fixed boundary condition, and is expressed as

$$
\Theta_{n}=C_{n} \sin (P x)
$$

where the parameter $P$ depends on the selected boundary condition. For a C-C, C-F, C-T, this parameter is defined as

$$
\begin{gathered}
P=n \pi / l \\
P=(2 n-1) \pi / 2 l \\
P=\alpha / l
\end{gathered}
$$

where $\alpha$ is determined by solving the following equation [91]

$$
\tan \alpha=-\alpha\left(G I_{\Phi} / k_{b} l\right)
$$

In the last relation, $k_{b}$ denotes the stiffness of the boundary spring. By substitution of Equation (29) into Equation (28) we obtain the following expression for the natural frequency

$$
\omega_{n}=\sqrt{\frac{G I_{\Phi} P^{2}}{\rho I_{\psi} P^{2}+I_{0}+\mu^{2}\left(I_{0} P^{2}+\rho I_{\psi} P^{4}\right)}}
$$

which can be redefined in dimensionless form as

$$
\bar{\omega}_{n}=\omega_{n} \times l \times \sqrt{\frac{\rho}{G}}
$$




\section{Results and Discussion}

This section is devoted to the numerical study of the vibration response for elliptical nanorods, with shear modulus $G=498 \mathrm{GPa}$ and density $\rho=1330 \mathrm{~kg} / \mathrm{m}^{3}$, in agreement with references [91-94]. A parametric investigation aims at checking the sensitivity of the response to some input parameters. The analysis starts with a comparative analysis of the first four dimensionless natural frequencies, for the reference circular case, under the assumptions $a=b=1 \mathrm{~nm}, \mu / l=0.1$ and $l=20 \mathrm{~nm}$, where we verify the accuracy of our results with respect to the available literature [95]. Then we consider the double effect of a varying geometrical ratio $b / a$ between the vertical and horizontal radius, and nonlocal parameter $\mu$, on a nanorod of length $l=20 \mathrm{~nm}$ with C-C (Table 1) or C-F (Table 2) boundary conditions. Based on the results from both tables, an increased nonlocal parameter yields a meaningless reduction of the natural frequency. At the same time, for a fixed nonlocal parameter, an increased dimensionless ratio $b / a$ gets a non monotonic variation of the natural frequencies, for both the selected boundary conditions. An increased frequency is noticed, first, for an increasing $b / a$ ratio from 0.1 up to 1 (i.e., when the elliptic shape reverts to the circular one). The contrary occurs for $b / a$ ratios higher than the unit value, with an overall decrease in the vibrational frequency of the nanostructure. In this last case, the elliptical shape becomes vertical, and the natural frequency reduces due to the decreased momentum of inertia compared to the horizontal elliptical shape, in agreement with Equation (26).

Table 1. Comparative evaluation of the first four dimensionless natural frequencies for a C-C circular nanorod with respect to the literature $(a=b=1 \mathrm{~nm}, \mu / l=0.1, l=20 \mathrm{~nm})$.

\begin{tabular}{ccccc}
\hline & $\mathbf{n = 1}$ & $\mathbf{n = 2}$ & $\mathbf{n}=\mathbf{3}$ & $\mathbf{n = 4}$ \\
\hline Present & 2.9971 & 5.3201 & 6.8586 & 7.8247 \\
Ref. [95] & 2.9971 & 5.3201 & 6.8586 & 7.8247 \\
\hline
\end{tabular}

Table 2. Free torsional vibration of C-C elliptical nanorod with a varying $b / a$ ratio and nonlocal parameter $\mu$.

\begin{tabular}{cccccc}
\hline $\boldsymbol{\mu} \times \mathbf{1 0}^{-\mathbf{9}}$ & $\boldsymbol{b} / \boldsymbol{a}=\mathbf{0 . 1}$ & $\boldsymbol{b} / \boldsymbol{a}=\mathbf{0 . 2}$ & $\boldsymbol{b} / \boldsymbol{a}=\mathbf{1}$ & $\boldsymbol{b} / \boldsymbol{a}=\mathbf{5}$ & $\boldsymbol{b} / \boldsymbol{a}=\mathbf{1 0}$ \\
\hline 0 & 0.6220 & 1.2082 & 3.1415 & 1.2062 & 0.6208 \\
1 & 0.6145 & 1.1935 & 3.1035 & 1.1916 & 0.6133 \\
1.5 & 0.6055 & 1.1760 & 3.0578 & 1.1741 & 0.6043 \\
2 & 0.5934 & 1.1526 & 2.9971 & 1.1508 & 0.5923 \\
\hline
\end{tabular}

A further physically consistent justification for this behavior is the fact that a twisted horizontal elliptical cross section features a lower angular rotation at a certain lapse of time than a vertical cross section due its higher stiffness.

Moreover, for both boundary conditions, the frequency response is symmetric with respect to the circular limit case (i.e., when $b / a=1$ ), with a non linear variation for both boundary conditions. According to a comparative evaluation of results in Tables 2 and 3, C-C nanorods yield higher frequencies than C-F structures, for the same fixed geometry and nonlocal assumptions. A C-C structure, indeed, is expected to exhibit a stiffer behavior than a C-F one, because of the intrinsically different nature of the two boundary conditions.

Table 3. Free torsional vibration of C-F elliptical nanorod with a varying $b / a$ ratio and nonlocal parameter $\mu$.

\begin{tabular}{cccccc}
\hline $\boldsymbol{\mu} \times \mathbf{1 0}^{-\mathbf{9}}$ & $\boldsymbol{b} / \boldsymbol{a}=\mathbf{0 . 1}$ & $\boldsymbol{b} / \boldsymbol{a}=\mathbf{0 . 2}$ & $\boldsymbol{b} / \boldsymbol{a}=\mathbf{1}$ & $\boldsymbol{b} / \boldsymbol{a}=\mathbf{5}$ & $\boldsymbol{b} / \boldsymbol{a}=\mathbf{1 0}$ \\
\hline 0 & 0.3110 & 1.2565 & 1.5708 & 1.2564 & 0.3108 \\
1 & 0.3100 & 1.2527 & 1.5659 & 1.2525 & 0.3099 \\
1.5 & 0.3089 & 1.2479 & 1.5600 & 1.2478 & 0.3087 \\
2 & 0.3072 & 1.2413 & 1.5517 & 1.2412 & 0.3071 \\
\hline
\end{tabular}


All the previous results are plotted in Figures 5 and 6, for a C-C and C-F boundary condition, respectively, where it is clearly visible that frequency computations based on a circular simplified assumption always overestimate the actual response of a more complicated actual geometrical cross section. All the plots in both figures, indeed, reach the peak value in the circular case (i.e., for $b / a=1$ ), which in turn, would be overestimated by a classical theory (i.e., for $\mu=0$ ), compared to a nonlocal theory. This means that possible nonlocalities within nanostructures must be properly estimated (also from an experimental standpoint) in order to provide accurate and physically consistent results.

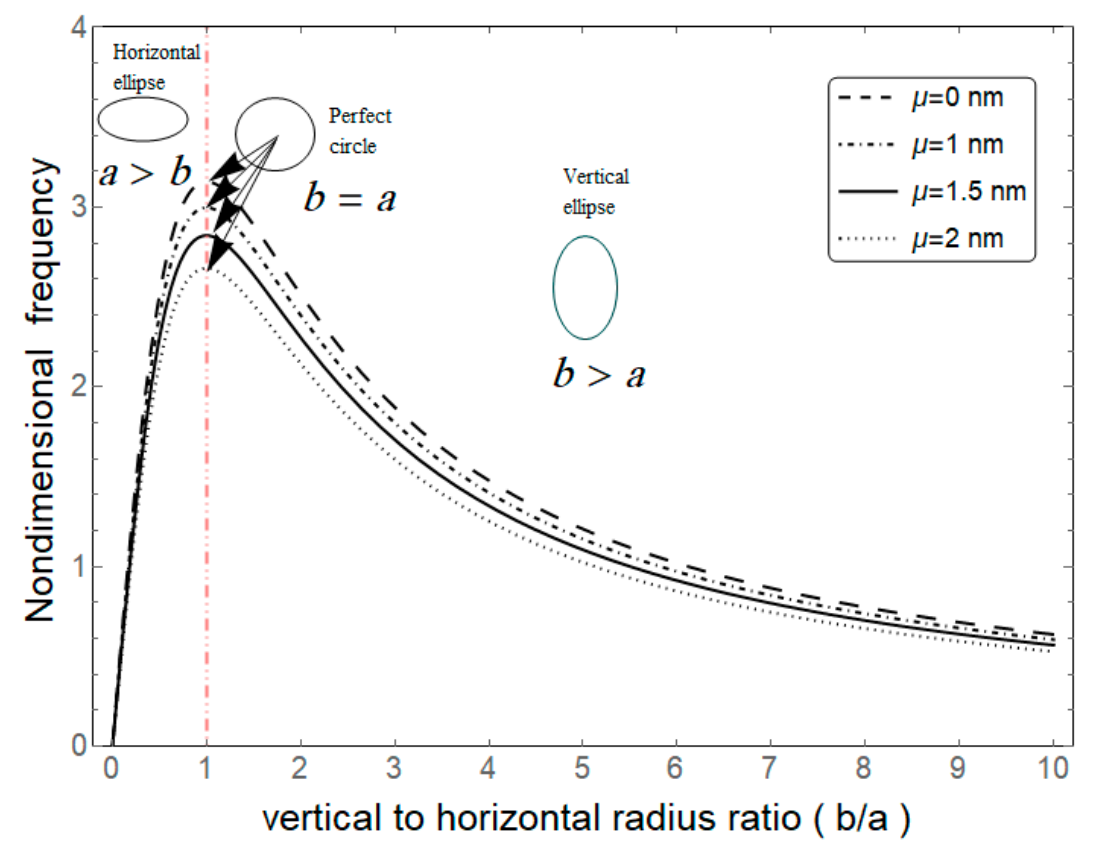

Figure 5. Variation of the nondimensional frequency ratio with the geometrical ratio $b / a$ for a C-C elliptical nanorod.

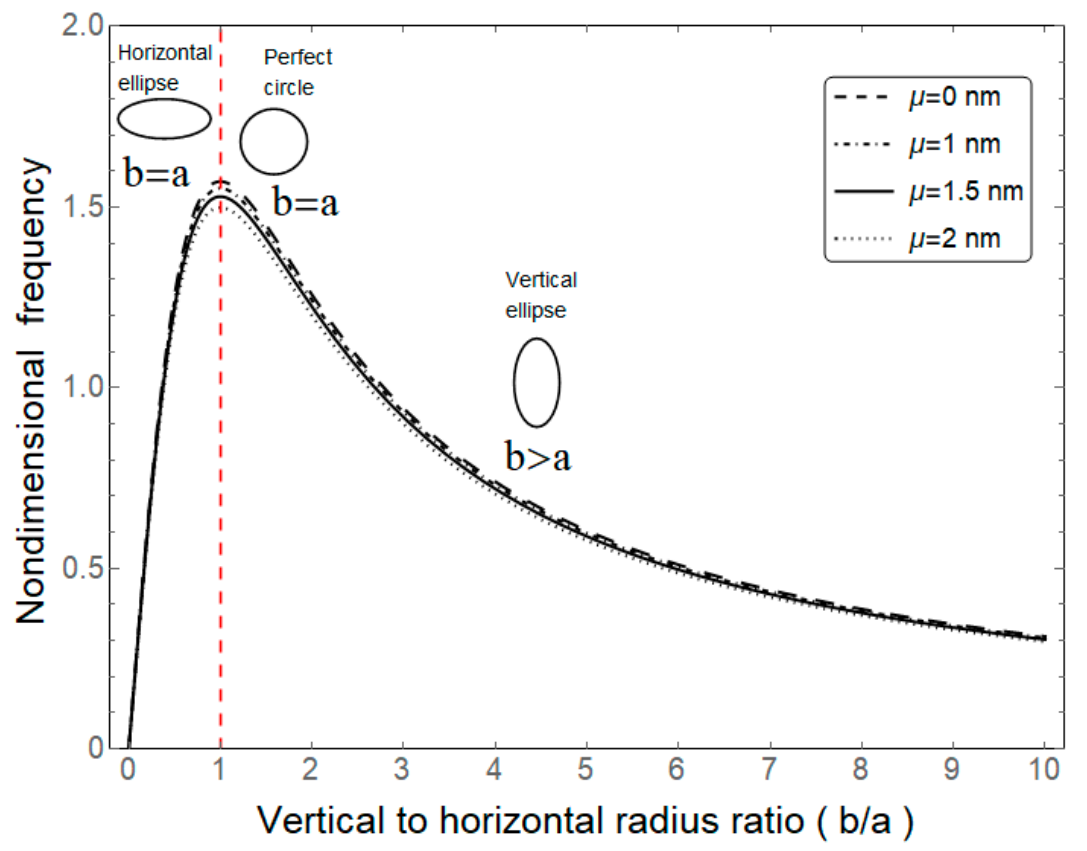

Figure 6. Variation of the nondimensional frequency ratio with the geometrical ratio $b / a$ for a C-F elliptical nanorod. 
A further systemic study considers the combined effect of the nonlocal parameter and stiffness of the boundary spring $\left(k_{b}\right)$ on the vibration response of a C-T elliptical nanorod with $a=0.4 \mathrm{~nm}$ and $b=0.2 \mathrm{~nm}$ (i.e., $b / a=0.5$ ), as summarized in Table 4 , and plotted in Figure 7 . Based on the results, it is worth noticing that increased torsional stiffness of $k_{b}$ from $0.01 \times 10^{-18}$ to $10 \times 10^{-18} \mathrm{GPa} \times \mathrm{nm}^{3}$ enables higher frequencies for the same fixed nonlocality of the structure, which means a reduction in the structural deformability. The sensitivity of the response to $k_{b}$ is more pronounced for lower values of the nonlocal parameter, and reduces gradually for higher nonlocalities. Moreover, a clear decrease in the natural frequency is observed, once again, for an increased nonlocality of the nanostructure, while keeping fixed the torsional stiffness of the boundary spring. This reflects the great importance of a correct modeling of boundary conditions for design purposes.

Table 4. Free torsional vibration of a C-T elliptical nanorod for a varying $\mu$ and $k_{b} . a=0.4 \mathrm{~nm}$, $b=0.2 \mathrm{~nm}$, and $l=20 \mathrm{~nm}$.

\begin{tabular}{ccccc}
\hline \multirow{2}{*}{$\boldsymbol{k}_{\boldsymbol{b}} \times \mathbf{1 0}^{-\mathbf{1 8}}$} & \multicolumn{4}{c}{$\boldsymbol{\mu} \times \mathbf{1 0}^{-\mathbf{9}}$} \\
\cline { 2 - 5 }$\left(\mathbf{G P a} \times \mathbf{n m}^{3}\right)$ & $\mathbf{0}$ & $\mathbf{1}$ & $\mathbf{1 . 5}$ & $\mathbf{2}$ \\
\hline 0.01 & 1.2815 & 1.2774 & 1.2723 & 1.2653 \\
0.1 & 1.4690 & 1.4628 & 1.4552 & 1.4448 \\
1 & 2.1225 & 2.1040 & 2.0817 & 2.0515 \\
10 & 2.4638 & 2.4351 & 2.4006 & 2.3547 \\
\hline
\end{tabular}

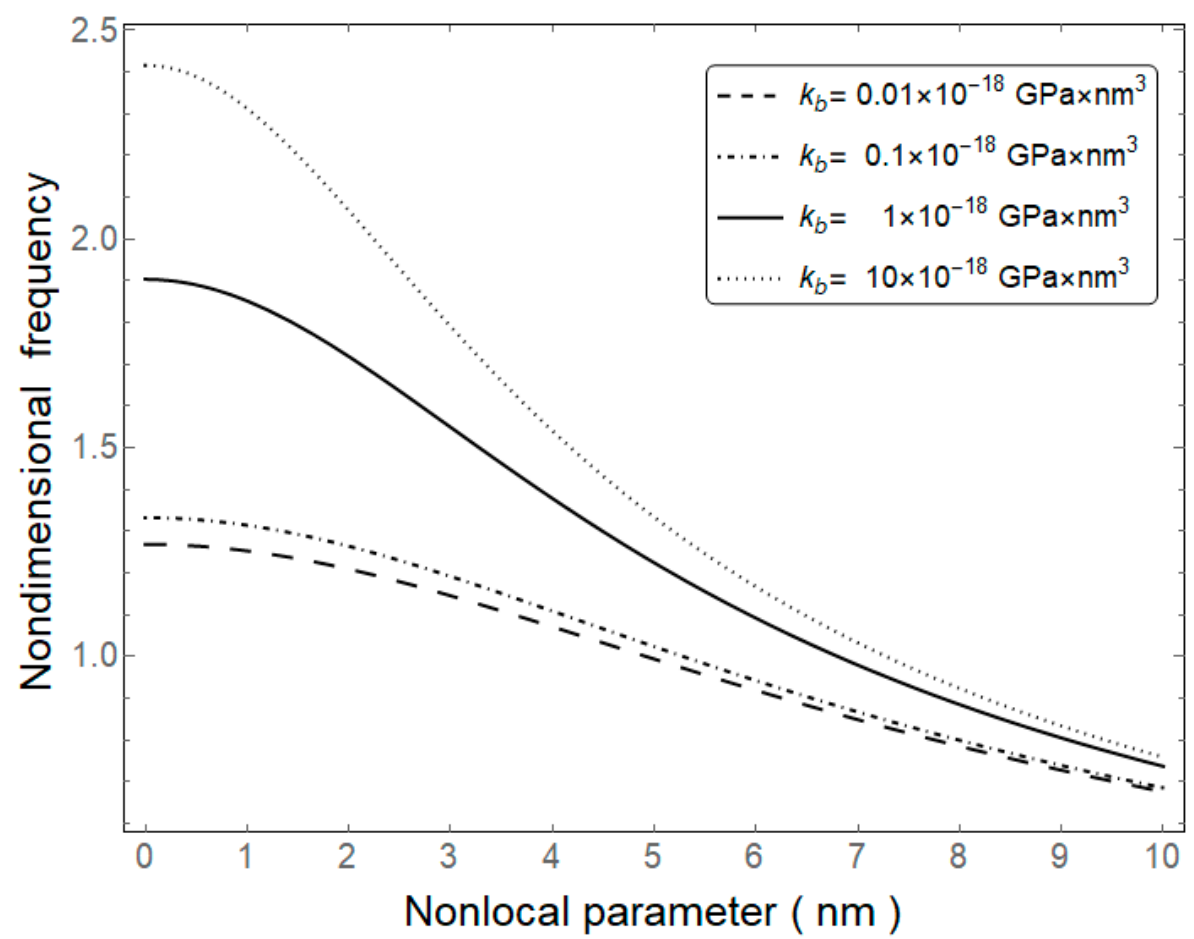

Figure 7. Variation of the nondimensional frequency ratio with $\mu$ and $k_{b} . a=0.4 \mathrm{~nm}, b=0.2 \mathrm{~nm}$, and $l=10 \mathrm{~nm}$.

\section{Concluding Remarks}

The free vibration of the torsional vibration of nanorods with an elliptical cross section is explored theoretically in this work, for three different boundary conditions, namely, a C-C, C-F, and C-T BCs. Hamilton's principle is selected to derive the equation of motion. A Galerkin method is here employed to solve the governing equation of the problem, where a parametric study is performed to check for the sensitivity of the vibration response to different parameters, including the nonlocal parameter, the geometrical vertical-to-horizontal $b / a$ radii, along with the boundary conditions. Based on the 
systematic investigation, it seems that an increased $b / a$ ratio up to the unit value exhibits increasing values of the frequency, while reaching the peak value for $b / a=1$. It follows a decreasing branch for $b / a>1$, due to the reduction of the momentum of inertia compared to a horizontal state of the ellipse. Moreover, an increased nonlocal parameter reduces the natural frequency, thus verifying the inaccuracy of classical theories compared to the nonlocal formulations. The structural response of nanorods is also affected by the selected boundary condition, where $\mathrm{C}-\mathrm{C}$ boundaries get higher frequencies, compared to a C-F boundary condition. In C-T nanorods, an increased stiffness of the spring provides higher natural frequencies, under the same fixed nonlocal assumptions. This sensitivity to the stiffness of the spring is more pronounced for lower values of the nonlocal parameter, and becomes meaningless for higher nonlocalities. A perfect circular shape always yields the highest natural frequencies, and could overestimate the actual twisting vibration response of a noncircular shape. These results are of great interest for design purposes, and could be extended to more complicated noncircular cross sections as a possible development of this work.

Author Contributions: Formal analysis, F.K., S.A.H., B.A.H., R.D. and F.T.; Investigation, F.K., S.A.H., B.A.H., R.D. and F.T.; Methodology, S.A.H., R.D. and F.T.; Software, F.K., F.T. and R.D.; Supervision, F.T.; Validation, B.A.H. and R.D.; Writing - original draft, F.K., S.A.H. and B.A.H.; Writing-review \& editing, R.D. and F.T. All authors have read and agreed to the published version of the manuscript.

Funding: This research received no external funding.

Conflicts of Interest: The authors declare no conflicts of interest.

\section{References}

1. Chopra, N.G.; Zettl, A. Measurement of the elastic modulus of a multi-wall boron nitride nanotube. Solid State Commun. 1998, 105, 297-300. [CrossRef]

2. Zhi, C.; Bando, Y.; Tang, C.; Golberg, D. Engineering of electronic structure of boron-nitride nanotubes by covalent functionalization. Phys. Rev. B 2006, 74, 153413. [CrossRef]

3. Wang, Z.L.; Dai, Z.R.; Gao, R.P.; Ba, Z.G. Side-by-side silicon carbide-silica biaxial nanowires: Synthesis, structure, and mechanical properties. Appl. Phys. Lett. 2000, 77, 3349. [CrossRef]

4. Latu-Romain, L.; Ollivier, M. Silicon carbide based one-dimensional nanostructure growth: Towards electronics and biology perspectives. J. Phys. D. Appl. Phys. 2014, 47, 203001. [CrossRef]

5. Krasavin, S.E.; Osipov, V.A. Vacancy-induced enhancement of thermal conductivity in grapheme. EPL (Europhys. Lett.) 2016, 113, 66002. [CrossRef]

6. Korobeynikov, S.N.; Alyokhin, V.V.; Babichev, A.V. On the molecular mechanics of single layer graphene sheets. Int. J. Eng. Sci. 2018, 133, 109-131. [CrossRef]

7. Salvetat, J.P.; Bonard, J.M.; Thomson, N.H.; Kulik, A.J.; Forrò, L.; Benoit, W.; Zuppiroli, L. Mechanical properties of carbon nanotubes. Appl. Phys. A 1999, 69, 255-260. [CrossRef]

8. Song, Y.W. Single-walled carbon nanotubes for high-energy optical pulse formation. Appl. Phys. Lett. 2008, 92, 021115. [CrossRef]

9. Kinoshita, Y.; Murashima, M.; Kawachi, M.; Ohno, N. First-principles study of mechanical properties of one-dimensional carbon nanotube intramolecular junctions. Comput. Mater. Sci. 2013, 70, 1-7. [CrossRef]

10. Férec, J.; Bertevas, E.; Khoo, B.C.; Ausias, G.; Phan-Thien, N. Thermal or electrical bulk properties of rod-filled composites. Int. J. Eng. Sci. 2018, 133, 219-230. [CrossRef]

11. Iijima, S. Helical microtubules of graphitic carbon. Nature 1991, 354, 56-58. [CrossRef]

12. Iijima, S.; Ichihashi, T. Single-shell carbon nanotubes of 1-nm diameter. Nature 1993, 363, 603-605. [CrossRef]

13. Baughman, R.H.; Cui, C.; Zakhidov, A.A.; Iqbal, Z.; Barisci, J.N.; Spinks, G.M.; Wallace, G.G.; Mazzoldi, A.; de Rossi, D.; Rinzler, A.G.; et al. Carbon nanotube actuators. Science 1999, 284, 1340-1344. [CrossRef]

14. Saito, Y.; Hta, K.; Tkakura, A.; Yotani, J.; Uemura, S. Field emission of carbon nanotubes and its application as electron sources of ultra-high luminance light-source devices. Physica B 2002, 323, 30-37. [CrossRef]

15. Wang, J. Carbon-nanotube based electrochemical biosensors: A review. Electroanal. An Int J. Dev. Fundam. Pract. Asp. Electroanal. 2005, 17,7-14. [CrossRef]

16. Arnold, M.S.; Green, A.A.; Hulvat, J.F.; Stupp, I.S.; Hersam, M.C. Sorting carbon nanotubes by electronic structure using density differentiation. Nat. Nanotech 2006, 1, 60-65. [CrossRef] 
17. Yan, J.; Zhou, H.; Yu, P. Rational functionalization of carbon nanotubes leading to electrochemical devices with striking applications. Adv. Mater. 2008, 20, 2899-2906. [CrossRef]

18. Eatemadi, A.; Daraee, H.; Karimkhanloo, H.; Kouhi, M.; Zarghami, N.; Akbarzadeh, A.; Abasi, M.; Hanifehpour, Y.; Joo, S.W. Carbon nanotubes: Properties, synthesis, purification, and medical applications. Nanoscale Res. Lett. 2014, 9, 393. [CrossRef] [PubMed]

19. Bianco, A.; Kostarelos, K.; Partidos, C.D.; Prato, M. Biomedical applications of functionalised carbon nanotubes. Chem. Commun. 2005, 5, 571-577. [CrossRef] [PubMed]

20. Miyako, E.; Hosokawa, C.; Kojima, M.; Yudasaka, M.; Funahashi, R.; Oishi, I.; Hagihara, Y.; Shichiri, M.; Takashima, M.; Nishio, K.; et al. A Photo-Thermal-Electrical Converter Based On Carbon Nanotubes for Bioelectronic Applications. Angew. Chem. Int. Ed. 2011, 50, 12266-12270. [CrossRef] [PubMed]

21. Gooding, J.J. Nanostructuring electrodes with carbon nanotubes: A review on electrochemistry and applications for sensing. Electrochim. Acta 2005, 50, 3049-3060. [CrossRef]

22. Paradise, M.; Goswami, T. Carbon nanotubes-production and industrial applications. Mat. Des. 2007, 28, 1477-1489. [CrossRef]

23. He, H.; Pham-Huy, L.A.; Dramou, P.; Xiao, D.; Zuo, P.; Pham-Huy, C. Carbon nanotubes: Applications in pharmacy and medicine. BioMed Res. Int. 2013, 578290. [CrossRef]

24. Braun, M.; Aranda-Ruiz, J.; Rodriguez-Millan, M.; Loya, J.A. On the bulk modulus and natural frequency of fullerene and nanotube carbon structures obtained with a beam based method. Compos. Struct. 2018, 187, $10-17$. [CrossRef]

25. Avouris, P.; Hertel, T.; Martel, R.; Schmidt, T.; Shea, H.R.; Walkup, R.E. Carbon nanotubes: Nanomechanics, manipulation, and electronic devices. Appl. Surf. Sci. 1999, 141, 201-209. [CrossRef]

26. Dai, H.; Hafner, J.H.; Rinzler, A.G.; Colbert, D.T.; Smalley, R.E. Nanotubes as nanoprobes in scanning probe microscopy. Nature 1996, 384, 147-150. [CrossRef]

27. Lau, A.K.T.; Hui, D. The revolutionary creation of new advanced materials-carbon nanotube composites. Compos. Part B Eng. 2002, 33, 263-277. [CrossRef]

28. Sun, C.H.; Li, F.; Cheng, H.M. Axial Young's modulus prediction of single-walled carbon nanotube arrays with diameters from nanometer to meter scales. Appl. Phys. Lett. 2005, 87, 193101. [CrossRef]

29. De Volder, M.F.; Tawfick, S.H.; Baughman, R.H.; Hart, A.J. Carbon nanotubes: Present and future commercial applications. Science 2013, 339, 535-539. [CrossRef]

30. Hibbeler, R.C. Statics and Mechanics of Materials, 4th Ed.; Prentice Hall: Upper Saddle River, NJ, USA, 2013.

31. Flügge, W. Tatik und Dynamik der Schalen; Springer: Berlin/Heidelberg, Germany, 2013.

32. Danesh, M.; Farajpour, A.; Mohammadi, M. Axial vibration analysis of a tapered nanorod based on nonlocal elasticity theory and differential quadrature method. Mech. Res. Commun. 2012, 39, 23-27. [CrossRef]

33. Malekzadeh, P.; Farajpour, A. Axisymmetric free and forced vibrations of initially stressed circular nanoplates embedded in an elastic medium. Acta Mech. 2012, 223, 2311-2330. [CrossRef]

34. Mohammadi, M.; Moradi, A.; Ghayour, M.; Farajpour, A. Exact solution for thermo-mechanical vibration of orthotropic mono-layer graphene sheet embedded in an elastic medium. Lat. Am. J. Solids Struct. 2014, 11, 437-458. [CrossRef]

35. Mohammadi, M.; Farajpour, A.; Goodarzi, M.; Dinari, F. Thermo-mechanical vibration analysis of annular and circular graphene sheet embedded in an elastic medium. Lat. Am. J. Solids Struct. 2014, 11, 659-682. [CrossRef]

36. Farajpour, A.; Rastgoo, A. Influence of carbon nanotubes on the buckling of microtubule bundles in viscoelastic cytoplasm using nonlocal strain gradient theory. Results Phys. 2017, 7, 1367-1375. [CrossRef]

37. Farajpour, A.; Ghayesh, M.H.; Farokhi, H. Large-amplitude coupled scale-dependent behaviour of geometrically imperfect NSGT nanotubes. Int. J. Mech. Sci. 2019, 150, 510-525. [CrossRef]

38. Farajpour, A.; Ghayesh, M.H.; Farokhi, H. A review on the mechanics of nanostructures. Int. J. Eng. Sci. 2018, 133, 231-263. [CrossRef]

39. Ghayesh, M.H.; Farajpour, A. A review on the mechanics of functionally graded nanoscale and microscale structures. Int. J. Eng. Sci. 2019, 137, 8-36. [CrossRef]

40. Eringen, A.C. On differential equations of nonlocal elasticity and solutions of screw dislocation and surface waves. J. Appl. Phys. 1983, 54, 4703-4710. [CrossRef] 
41. Eringen, A.C. Plane waves in nonlocal micropolar elasticity. Int. J. Eng. Sci. 1984, 22, 1113-1121. [CrossRef]

42. Eringen, A.C. Nonlocal polar elastic continua. Int. J. Eng. Sci. 1972, 10, 1-16. [CrossRef]

43. Eringen, A.C. Nonlocal Continuum Field Theories; Springer Science \& Business Media: Berlin, Germany, 2002.

44. Eringen, A.C.; Edelen, D. On nonlocal elasticity. Int. J. Eng. Sci. 1972, 10, 233-248. [CrossRef]

45. Sudak, L.J. Column buckling of multiwalled carbon nanotubes using nonlocal continuum mechanics. J. Appl. Phys. 2003, 94, 7281-7287. [CrossRef]

46. Li, C.Y.; Chou, T.W. Vibrational behaviors of multi-walled carbon nanotube-based nanomechancial resonators. Appl. Phys. Lett. 2004, 84, 121-123. [CrossRef]

47. He, X.Q.; Kitipornchai, S.; Liew, K.M. Buckling analysis of multi-walled carbon nanotubes: A continuum model accounting for van der Waals interaction. J. Mech. Phys. Solid 2005, 53, 303-326. [CrossRef]

48. Zhang, Y.Q.; Liu, G.R.; Xie, X.Y. Free transverse vibrations of double-walled carbon nanotubes using a theory of nonlocal elasticity. Phys. Rev. B 2005, 71, 195404. [CrossRef]

49. Wang, Q.; Varadan, V.K. Vibration of carbon nanotubes studied using nonlocal continuum mechanics. Smart Mat. Struct. 2006, 15, 659-666. [CrossRef]

50. Aranda-Ruiz, J.; Loya, J.A.; Fernández-Sáez, J. Bending vibrations of rotating nonuniform nanocantilevers using the Eringen nonlocal elasticity theory. Compos. Struct. 2012, 94, 2990-3001. [CrossRef]

51. Numanoğlu, H.M.; Akgöz, B.; Civalek, Ö. On dynamic analysis of nanorods. Int. J. Eng. Sci. 2018, 130, 33-50. [CrossRef]

52. Malikan, M.; Dimitri, R.; Tornabene, F. Transient response of oscillated carbon nanotubes with an internal and external damping. Compos. Part B-Eng. 2019, 158, 198-205. [CrossRef]

53. Jalaei, M.H.; Civalek, Ö. On dynamic instability of magnetically embedded viscoelastic porous FG nanobeam. Int. J. Eng. Sci. 2019, 143, 14-32. [CrossRef]

54. Numanoğlu, H.M.; Civalek, Ö. On the dynamics of small-sized structures. Int. J. Eng. Sci. 2019, 145, 103164. [CrossRef]

55. Lim, C.W. Equilibrium and static deflection for bending of a nonlocal nanobeam. Adv. Vib. Eng. 2009, 8, 277-300.

56. Kiani, K.; Mehri, B. Assessment of nanotube structures under a moving nanoparticle using nonlocal beam theories. J. Sound Vib. 2010, 329, 2241-2264. [CrossRef]

57. Lim, C.W.; Niu, J.C.; Yu, Y.M. Nonlocal stress theory for buckling instability of nanotubes: New predictions on stiffness strengthening effects of nanoscales. J. Comput. Theor. Nanosci. 2010, 7, 2104-2111. [CrossRef]

58. Li, C.; Lim, C.W.; Yu, J.L. Dynamics and stability of transverse vibrations of nonlocal nanobeams with a variable axial load. Smart Mat. Struct. 2011, 20, 015023. [CrossRef]

59. Fernández-Sáez, J.; Zaera, R.; Loya, J.A.; Reddy, J.N. Bending of Euler-Bernoulli beams using Eringen's integral formulation: A paradox resolved. Int. J. Eng. Sci. 2016, 99, 107-116. [CrossRef]

60. Demir, Ç.; Civalek, Ö. On the analysis of microbeams. Int. J. Eng. Sci. 2017, 121, 14-33. [CrossRef]

61. Jouneghani, F.Z.; Dimitri, R.; Tornabene, F. Structural response of porous FG nanobeams under hygro-thermo-mechanical loadings. Compos. Part B Eng. 2018, 152, 71-78. [CrossRef]

62. Arefi, M.; Bidgoli, E.M.R.; Dimitri, R.; Bacciocchi, M.; Tornabene, F. Nonlocal bending analysis of curved nanobeams reinforced by graphene nanoplatelets. Compos. Part B Eng. 2019, 166, 1-12. [CrossRef]

63. Pinnola, F.P.; Faghidian, S.A.; Barretta, R.; de Sciarra, F.M. Variationally consistent dynamics of nonlocal gradient elastic beams. Int. J. Eng. Sci. 2020, 149, 103220. [CrossRef]

64. Duan, W.H.; Wang, C.M. Exact solutions for axisymmetric bending of micro/nanoscale circular plates based on nonlocal plate theory. Nanotechnology 2007, 18, 385704. [CrossRef]

65. Murmu, T.; Pradhan, S.C. Vibration analysis of nanoplates under uniaxial prestressed conditions via nonlocal elasticity. J. Appl. Phys. 2009, 106, 104301. [CrossRef]

66. Aghababaei, R.; Reddy, J.N. Nonlocal third-order shear deformation plate theory with application to bending and vibration of plates. J. Sound Vib. 2009, 326, 277-289. [CrossRef]

67. Malikan, M.; Tornabene, F.; Dimitri, R. Nonlocal three-dimensional theory of elasticity for buckling behavior of functionally graded porous nanoplates using volume integrals. Mater. Res. Express 2018, 5, 095006. [CrossRef]

68. Srividhya, S.; Raghu, P.; Rajagopal, A.; Reddy, J.N. Nonlocal nonlinear analysis of functionally graded plates using third-order shear deformation theory. Int. J. Eng. Sci. 2018, 125, 1-22. [CrossRef] 
69. Dindarloo, M.H.; Li, L.; Dimitri, R.; Tornabene, F. Nonlocal elasticity response of doubly-curved nanoshells. Symmetry 2020, 12, 466. [CrossRef]

70. Malikan, M.; Krasheninnikov, M.; Eremeyev, V.A. Torsional stability capacity of a nano-composite shell based on a nonlocal strain gradient shell model under a three-dimensional magnetic field. Int. J. Eng. Sci. 2020, 148, 103210. [CrossRef]

71. Malikan, M.; Eremeyev, V.A. Free Vibration of Flexomagnetic Nanostructured Tubes Based on Stress-Driven Nonlocal Elasticity, In Analysis of Shells, Plates, and Beams; Springer: Berlin/Heidelberg, Germany, 2020; pp. 215-226, Chapter 12.

72. Ansari, R.; Gholami, R.; Ajori, S. Torsional vibration analysis of carbon nanotubes based on the strain gradient theory and molecular dynamic simulations. J. Vib. Acoustic. 2013, 135, 051016. [CrossRef]

73. Fatahi-Vajari, A.; Imam, A. Torsional vibration of single-walled carbon nanotubes using doublet mechanics. Z. Angew. Math. Phys. 2016, 67, 81. [CrossRef]

74. Arda, M.; Aydogdu, M. Torsional vibration of double CNT system embedded in an elastic medium. Noise Theory Pract. 2018, 4, 4 .

75. Li, C. Torsional vibration of carbon nanotubes: Comparison of two nonlocal models and a semi-continuum model. Int. J. Eng. Sci. 2014, 82, 25-31. [CrossRef]

76. Aydogdu, M.; Arda, M. Torsional vibration analysis of double walled carbon nanotubes using nonlocal elasticity. Int. J. Mech. Mater. Design 2016, 12, 71-84. [CrossRef]

77. Demir, C.; Civalek, Ö. Torsional and longitudinal frequency and wave response of microtubules based on the nonlocal continuum and nonlocal discrete models. Appl. Math. Model. 2013, 37, 9355-9367. [CrossRef]

78. Murmu, T.; Adhikari, S.; Wang, C. Torsional vibration of carbon nanotube-buckyball systems based on nonlocal elasticity theory. Phys. E Low Dimens. Syst. Nanostruct. 2011, 43, 1276-1280. [CrossRef]

79. Suzuki, N.; Yokoi, H.; Shamoto, E. Micro/nano sculpturing of hardened steel by controlling vibration amplitude in elliptical vibration cutting. Precis. Eng. 2011, 35, 44-50. [CrossRef]

80. Zhang, J.; Cui, T.; Ge, C.; Sui, Y.; Yang, H. Review of micro/nano machining by utilizing elliptical vibration cutting. Int. J. Mach. Tool Manufact. 2016, 196, 109-126. [CrossRef]

81. Yang, Y.; Pan, Y.; Guo, P. Structural coloration of metallic surfaces with micro/nano-structures induced by elliptical vibration texturing. Appl. Surf. Sci. 2017, 402, 400-409. [CrossRef]

82. Mikeš, K.; Jirásek, M. Free warping analysis and numerical implementation. Appl. Mech. Mat. 2016, 825, 141-148. [CrossRef]

83. Barr, A. Torsional waves in uniform rods of non-circular section. J. Mech. Eng. Sci. 1962, 4, 127-135. [CrossRef]

84. Stephen, N. Comparison of dynamic torsion theories for beams of elliptical cross-section. J. Sound Vib. 1985, 100, 1-6. [CrossRef]

85. Francu, J.; Novácková, P.; Janicek, P. Torsion of a non-circular bar. Eng. Mech. 2012, 19, 45-60.

86. Christides, S.; Barr, A. Torsional vibration of cracked beams of non-circular cross-section. Int. J. Mech. Sci. 1986, 28, 473-490. [CrossRef]

87. Loya, J.A.; Aranda-Ruiz, J.; Fernández-Sáez, J.F. Torsion of cracked nanorods using a nonlocal elasticity model. J. Phys. D Appl. Phys. 2014, 47, 115304. [CrossRef]

88. Loya, J.; López-Puente, J.; Zaera, R.; Fernández-Sáez, J. Free transverse vibrations of cracked nanobeams using a nonlocal elasticity model. J. Appl. Phys. 2009, 105, 044309. [CrossRef]

89. Loya, J.A.; Rubio, L.; Fernández-Sáez, J. Natural frequencies for bending vibrations of Timoshenko cracked beams. J. Sound Vib. 2006, 290, 640-653. [CrossRef]

90. Ogata, Y.; Mizutani, G. Control of cross-sections and optical nonlinearity of Pt Nanowires and the Roughness Effect. Phys. Res. Int. 2012, 2012, 969835. [CrossRef]

91. Rao, S.S. Vibration of Continuous Systems; Wiley Online Library: Hoboken, NJ, USA, 2007.

92. Mir, M.; Hosseini, A.; Majzoobi, G. A numerical study of vibrational properties of single-walled carbon nanotubes. Comp. Mater. Sci. 2008, 43, 540-548. [CrossRef]

93. Ruoff, R.S.; Qian, D.; Liu, W.K. Mechanical properties of carbon nanotubes: Theoretical predictions and experimental measurements. Comptes. Rendus. Phys. 2003, 4, 993-1008. [CrossRef] 
94. El Khoury, E.; Messager, T.; Cartraud, P. Derivation of the Young's and shear moduli of single-walled carbon nanotubes through a computational homogenization approach. Int. J. Multisc. Comput. Eng. 2011, 9, 97-118. [CrossRef]

95. Khosravi, F.; Hosseini, S.A.; Tounsi, A. Torsional dynamic response of viscoelastic SWCNT subjected to linear and harmonic torques with general boundary conditions via Eringen's nonlocal differential model. Eur. Phys. J. Plus 2020, 135, 183. [CrossRef] 